\title{
Acute non-renal adverse events after unenhanced and enhanced computed tomography and magnetic resonance imaging
}

\author{
Manal Azzouz ${ }^{1}$, Janne Rømsing ${ }^{2}$, Henrik S. Thomsen ${ }^{1}$ \\ ${ }^{1}$ Department of Diagnostic Radiology, Copenhagen University Hospital Herlev, Herlev, Denmark \\ ${ }^{2}$ Department of Drug Design and Pharmacology, Faculty of Health and Medical Sciences, Copenhagen, Denmark \\ Email: manalazzouz@gmail.com
}

Received 24 May 2013; revised 24 June 2013; accepted 1 July 2013

Copyright (C) 2013 Manal Azzouz et al. This is an open access article distributed under the Creative Commons Attribution License, which permits unrestricted use, distribution, and reproduction in any medium, provided the original work is properly cited.

\section{ABSTRACT}

Aim: Investigating the incidence of non-renal acute adverse events (AAEs) in patients undergoing unenhanced or enhanced computed tomography (CT) or magnetic resonance (MR) (four groups) using the active interview-method. Methods: The imaging procedure followed the standards of the department. Furthermore, the iodine-based (nonionic monomer) and gadolinium-based (cyclic) contrast agent was the same as used for all other examinations in the department. All patients were interviewed for $\mathbf{7 2}$ hours after the MR or CT examination regarding occurrence of AAEs using a structured questionnaire. Results: A total of 1358 patients (259 with enhanced MR, 434 with unenhanced MR, 450 with enhanced CT and 215 with unenhanced CT) were enrolled. AAEs were significantly higher $(P<0.05)$ in the contrast enhanced MR [48 (18.5\%)] and CT [35 (7.7\%)] groups compared to unenhanced MR [39 (9.0\%)] and CT [5 (2.3\%)] groups. Dizziness was significantly [19 (7.3\%), $(P<0.05)]$ more frequent in the enhanced MR. The same AAEs were reported in both contrast groups and control groups. Conclusion: AAEs which are mainly considered to be caused by the contrast medium are also experienced by patients undergoing CT or MR without contrast. Enhanced MR is correlated with more reactions than unenhanced MR and enhanced CT. Dizziness was reported more frequently after MR than $\mathrm{CT}$, both with and without contrast; more patients were CNS examined with MR and with CT.

Keywords: Acute Adverse Events; Contrast Media; Gadolinium-Based Contrast Media; Iodine-Based Contrast Media

\section{INTRODUCTION}

For many years, it has been debated whether all the nonrenal acute adverse events (AAE) occurring after administration of contrast media really are caused by the agent [1]. Could they be caused by other factors? Lalli [2] believed that it is possible to explain all reactions to contrast media through CNS mechanisms. Weber [3] showed that, when a new drug is introduced to the market, adverse reactions related to the drug are over-reported. This indicates that all reactions may not necessarily be due to the agent in all instances.

In radiology a randomized study involving an arm, where the patients receive contrast medium, and an arm, where a substance with no active molecule but similar to the agent (smell, vial, osmolality, viscosity) is administered, is difficult to perform; it requires that the person who administers the agent does not participate in imaging procedure. Furthermore, it would be unethical to randomize a patient with e.g. liver metastases to either contrast medium or no contrast medium.

Nearly all studies of the incidence of non-renal AAEs deals with a single agent with no control group or compare two agents. Therefore we undertook a prospective study of AAEs in patients undergoing 1) enhanced computed tomography (CT), 2) unenhanced CT, 3) enhanced magnetic resonance (MR) or 4) unenhanced MR.

\section{MATERIAL AND METHODS}

This study was approved by the Local Committee for Health Research Ethics (No. H-1-2010-011) and the Danish Data Protection Agency. It was registered at Clinical-Trials.gov (No. NCT01132339). Written informed consent was obtained from all patients who participated in the study. 


\subsection{Patients}

Adults scheduled to undergo CT or MR examination with or without administration of contrast medium, were eligible to participate. The exclusion criteria were as follow: patients younger than 18 years old, patients diagnosed with hyperthyroidism (only CT patients) or dementia, pregnant or lactating women, patients that had surgery 30 days before the examination, patients that had undergone enhanced or unenhanced CT or MR examination within the past 4 weeks, if further enhanced or unenhanced CT or MR examination were planned or expected during the 3 days observation period and if patients were unable to complete their CT or MR examination.

\subsection{Sampling Method}

The patients were referred to a CT or MR examination as part of their work-up due to signs and symptoms of various diseases, and were therefore allocated to one of the four study groups without any involvement of the study investigator. The patients were allocated on pure clinical indications to receive either contrast enhanced CT with intravenous iodine-based (nonionic monomer) contrast medium, or contrast enhanced MR with intravenous gadolinium-based (ionic cyclic) contrast medium, or CT or MR examination without contrast medium. The groups which did not receive contrast medium are referred to as control groups.

\subsection{Contrast Media}

CT and MR examination were performed according to the department's protocols.

\subsubsection{Iodine-Based Contrast Medium}

For CT examinations a low osmolar non-ionic contrast medium iomeprol 350 - 400 mg I/ml (Iomeron; Bracco, Milan, Italy) was administrated for examination of: head, spine, thorax/abdomen, extremity. The osmolality of iomeprol solution of $350 \mathrm{mg} \mathrm{I} / \mathrm{ml}$ is $618 \mathrm{mOsmol}$. The dose administered varied between 70 and $155 \mathrm{ml}$.

\subsubsection{Gadolinium-Based Contrast Medium}

For MR examinations a macrocyclic contrast medium gadoterate meglumine (Dotarem; Guerbet, Roissy CDG, France) was given for the following examination: head, spine, thorax, abdomen, pelvis, extremity and whole body. The osmolality of gadoteric acid is $1350 \mathrm{mOsm} / \mathrm{kg}$ and content is $279.3 \mathrm{mg} / \mathrm{ml}$. Standard dose for all examinations was $0.2 \mathrm{ml} / \mathrm{kg}$.

\subsection{Data Collection}

According to departments protocol the patients left the hospital immediately after the CT/MR examination. Therefore all patients were contacted 72 hours after the examination and interviewed according to a structured questionnaire (Figure 1) for occurrence of AAEs after contrast media administration if injection were required, or after CT/MR examination start. The severity of AAEs was sub-classified into mild, moderate and severe acute non-renal adverse events.

\subsection{Statistical Analysis}

Demographic information and AAEs were analyzed using descriptive statistics. Age and body mass index were compared with the Mann-Whitney U test between contrasts enhanced and control groups. The two-sided Fisher exact test was used to analyze the proportion of AAEs, and the proportion of a specific AAE experienced between the enhanced CT and MR and control groups. A p-value of 0.05 or less was considered to indicate a statistically significant difference. All statistical analyses were performed using IBM SPSS Statistics software, (version 20.0; IBM Corp).

\section{RESULTS}

From September 2010 to December 2012, a total of 1358 patients were included in the study (Table 1). More than 400 patients were included in the MR control and the contrast enhanced CT groups. The patients in the contrast enhanced MR group were younger $(P=0.008)$ than in the MR control group whereas the opposite was the case for the two CT groups ( $\mathrm{P}=0.013)$. The patients in the control group weighed significantly more than those in the contrast enhanced MR $(\mathrm{P}=0.017)$ and CT $(\mathrm{P}<0.001)$ groups. In the MR groups a higher ratio of females to males was included, while in the CT groups the ratio of males is slightly higher to females. Patients in the contrast enhanced MR group had undergone renal surgery significantly more frequently than the patients in the control group $(\mathrm{P}=0.017)$, but with regards to presence of gout the opposite was observed $(\mathrm{P}<0.001)$. Patients in the CT control group reported significantly more frequent kidney disease, previous renal surgery and gout than the patients in the contrast enhanced CT group.

More than $80 \%$ of the patients in the contrast enhanced MR group underwent brain or abdominal MR whereas this was the case for only $20 \%$ in the MR control group $(\mathrm{P}<0.001)$. In the MR control group $78 \%$ of the patients underwent MR of an extremity, spine or whole body imaging. In the two CT groups, an overwhelming majority of patients underwent CT of the chest and/ or abdomen.

\section{Acute Adverse Events}

In the contrast enhanced MR group $15.4 \%$ of the patients 


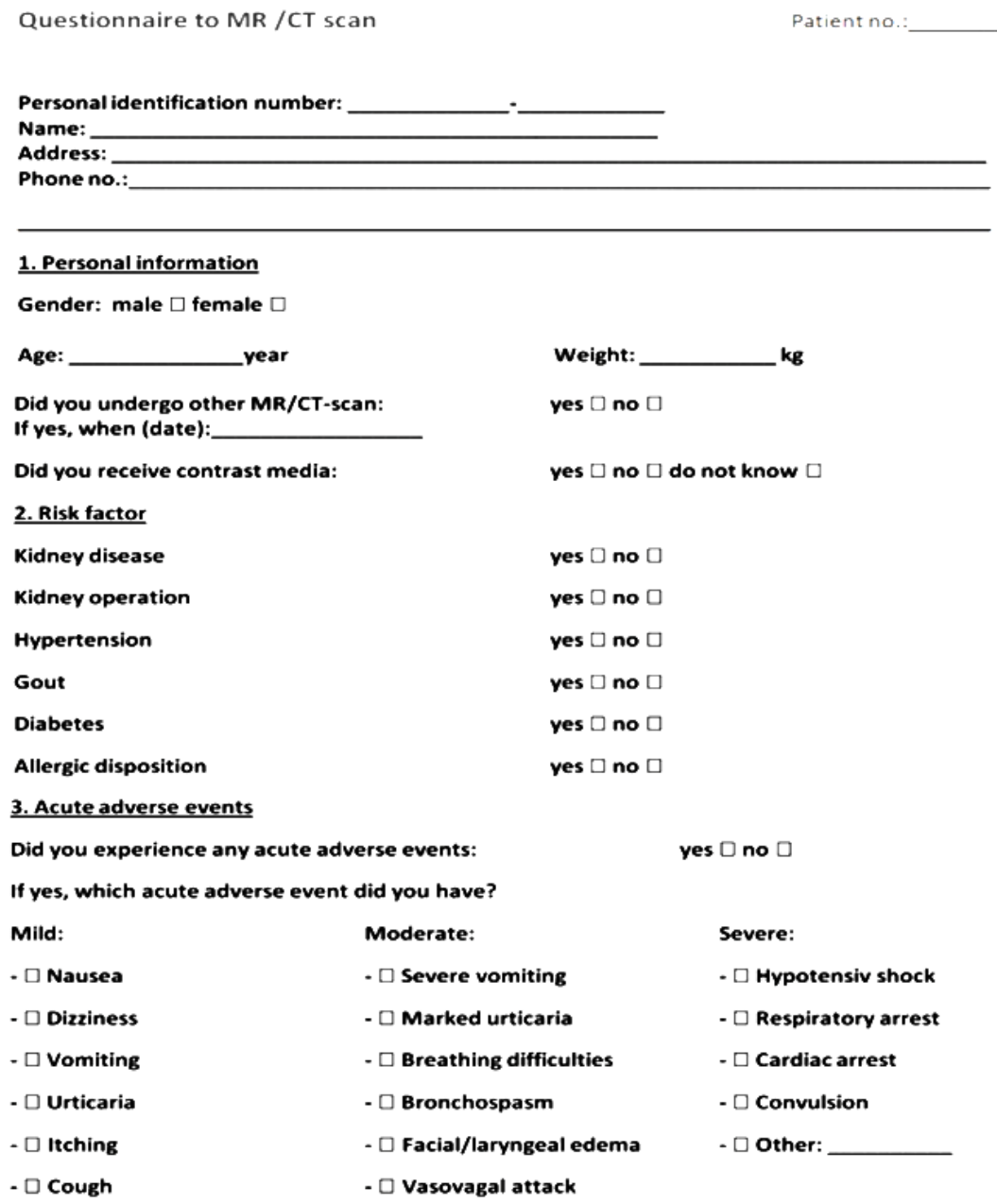

Figure 1. Questionnaire to MR/CT scan. Parts 1 and 2 (personal information and risk factors) were completed at time for enrollment before the MR/CT examination, and part 3 (occurrence of acute adverse events) was completed 72 hours later.

reported an AAE or described a symptom they developed, while it was significantly lower (7.4\%) in the MR control group ( $\mathrm{P}<0.001)$, (Table 2). Thirteen patients experienced more than one AAE. Dizziness was the most frequent adverse event in both groups, but it occurred significantly more frequently in the contrast enhanced MR group than in the control group $(\mathrm{P}=0.014)$. In the CT groups, the prevalence of AAEs was $5.8 \%$ in the contrast group and $1.9 \%$ in the control group $(\mathrm{P}=$ 0.026). Eight patients had more than one AAE. The most frequent reported AAEs were nausea and dizziness, but the difference between the two groups was insignificant (Table 3).

With one exception (marked urticaria in the contrast enhanced CT group), all reported AAEs were classified as mild. No severe adverse events occurred.

\section{DISCUSSION}

Studies of AAEs to contrast media do not, with one exception, include a control group. In 2006 a prospective randomized study reported by Schild et al. showed that the AAEs seen in the group administered with contrast medium intravenously also occurred in the control group undergoing the same imaging examination [4]. The incidence was, however; significantly lower in the control group (9.4\%) than in the monomeric contrast group (44.8\%). In accordance with Schild et al. [4] the incidence of AAEs in our study was significantly lower in the CT control group (1.9\%) compared to the contrast enhanced CT group (5.8\%), although the incidence of AAEs was lower in our study.

According to Lalli [3] other factors than the contrast medium introduces adverse events. With one exception, all reported adverse events in the present study were mild. This observation raises an important issue. When a mild AAE is observed, is it due to the agent or the circumstances? If it, incorrectly, is classified as a contrast induced adverse event the patient may be denied another 
Table 1. Descriptive data to MR and CT groups.

\begin{tabular}{|c|c|c|c|c|c|c|}
\hline Variable & $\begin{array}{c}\text { Contrast enhanced } \\
\text { MR n = } 259\end{array}$ & $\begin{array}{c}\text { MR control } \\
\mathrm{n}=434\end{array}$ & P-value & $\begin{array}{c}\text { Contrast enhanced } \\
\text { CT n }=450\end{array}$ & $\begin{array}{l}\text { CT control } \\
\mathrm{n}=215\end{array}$ & P-value \\
\hline $\operatorname{Age}(y)^{\S}$ & $52.9 \pm 17.5(18-96)$ & $56.7 \pm 14.6(23-89)$ & 0.008 & $65.3 \pm 10.9(18-88)$ & $61.9 \pm 14.3(18-91)$ & 0.013 \\
\hline Male/Female & $\begin{array}{l}106(40.9 \%) / \\
153(59.1 \%)\end{array}$ & $\begin{array}{l}154(35.5 \%) / \\
280(64.5 \%)\end{array}$ & 0.168 & $\begin{array}{l}233(51.7 \%) / \\
217(48.2 \%)\end{array}$ & $\begin{array}{c}128(59.5 \%) / \\
87(40.5 \%)\end{array}$ & 0.067 \\
\hline Weight $(\mathbf{k g})^{\S}$ & $\begin{array}{c}74.9 \pm 16.2 \\
(40-127)\end{array}$ & $\begin{array}{c}78.4 \pm 18.2 \\
(44-192)\end{array}$ & 0.017 & $\begin{array}{c}74.4 \pm 16.2 \\
(40-136)\end{array}$ & $\begin{array}{c}80.9 \pm 17.1 \\
(44-160)\end{array}$ & $<0.001$ \\
\hline \multicolumn{7}{|l|}{ Risk factors } \\
\hline Kidney disease & $15(5.8 \%)$ & $20(4.6 \%)$ & 0.480 & 39 (8.7\%) & 64 (29.8\%) & $<0.001$ \\
\hline Kidney operation & $13(5.0 \%)$ & $7(1.6 \%)$ & 0.017 & $43(9.6 \%)$ & $50(23.3 \%)$ & $<0.001$ \\
\hline Hypertension & $63(24.3 \%)$ & 132 (30.4\%) & 0.07 & $161(35.8 \%)$ & $83(38.6 \%)$ & 0.492 \\
\hline Gout & $60(23.2 \%)$ & $165(38.0 \%)$ & $<0.0001$ & 97 (21.6\%) & $64(29.8 \%)$ & 0.026 \\
\hline Diabetes & $16(6.2 \%)$ & $44(10.1 \%)$ & 0.093 & $55(12.2 \%)$ & $28(13.0 \%)$ & 0.802 \\
\hline Allergic disposition & $53(20.5 \%)$ & $98(22.6 \%)$ & 0.569 & $54(12.0 \%)$ & 38 (17.7\%) & 0.055 \\
\hline \multicolumn{7}{|l|}{ MR/CT type } \\
\hline Head & $130(50.2 \%)$ & $33(7.6 \%)$ & $<0.001$ & $5(1.1 \%)$ & 19 (8.8\%) & $<0.001$ \\
\hline Spine & $12(4.6 \%)$ & $122(28.1 \%)$ & $<0.001$ & $2(0.4 \%)$ & $3(1.4 \%)$ & 0.335 \\
\hline Chest & $4(1.5 \%)$ & $5(1.2 \%)$ & 0.734 & 0 & 0 & \\
\hline Abdomen & 79 (30.5\%) & 52 (11.9\%) & $<0.001$ & 0 & 0 & \\
\hline Chest/abdomen & 0 & 0 & & 443 (98.4\%) & $181(84.2 \%)$ & $<0.001$ \\
\hline Pelvis & $5(1.9 \%)$ & $2(0.5 \%)$ & 0.109 & 0 & 0 & \\
\hline Extremity & 29 (11.2\%) & 159 (36.6\%) & $<0.001$ & 0 & $12(5.6 \%)$ & $<0.001$ \\
\hline Whole body & $1(0.4 \%)$ & $61(14.1 \%)$ & $<0.001$ & 0 & 0 & \\
\hline
\end{tabular}

${ }^{\S}$ Data are means \pm standard deviation, with ranges in parentheses.

enhanced examination or recommended to use premedication on an incorrect background. In modern world a lot of data about the patients are registered, and the current study raises the question whether a mild AAE should be registered under the "warning" in the patient records or should only adverse events requiring treatment be listed.

The four groups were in general comparable with regard to age, sex, weight, whereas the disease pattern differed. The AAEs incidence rate was $15.4 \%$ in the contrast enhanced MR group and $7.4 \%$ in the MR control group, and $5.8 \%$ in the contrast enhanced CT group and $1.9 \%$ in the CT control group. This indicates that almost half of the $15.4 \%$ in the contrast enhanced MR group and one fourth part of the $5.8 \%$ in the contrast enhanced CT group of the AAEs incidence rate may not be related to the administration of contrast media. In other studies, the incidence of AAEs reported ranged from $0.017 \%$ to 2.4\% to contrast enhanced MR agents, and 3\% for nonionic low osmolar contrast enhanced CT agents [5-8].

The high incidence of AAEs found in both the MR and CT groups in our study may be because, all information about presence of AAEs were obtained through an active interview using a structured questionnaire. The effect of an active interview on frequency of AAEs to contrast media has been investigated in 863 patients by Thomsen [9]. AAEs were only reported in the interviewed group with an incidence rate of $1.9 \%$, while the non-interviewed group did not report any AAEs. However, they were asked whether they felt anything in relation to the injection of the contrast agent. The patients underwent MR in a silent 0.1T MR scanner. In our study the patients were contacted 72 hours later for occurrence of AAEs and a structured questionnaire was used (Figure 1). This is probably the explanation for the different incidence (15.4 vs. $1.9 \%$ ) in the two studies. According to Lalli [3], the most important factors in triggering a contrast media reaction are the patients fear and anxiety, through CNS mechanisms. This factor combined with an active interview of the patients may have increased the frequency of AAEs reported in our study.

It is a general understanding that AAEs occur more frequently after iodine-based contrast media than after gadolinium-based contrast media [6,10,11]. Hunt et al. [12] showed in a retrospective study of 456.930 contrast doses, that the incidence of adverse effects was higher with low-osmolar iodinated contrast media (0.15\%) than with gadolinium contrast media $(0.04 \%)$ [12]. In our study, more patients undergoing MR reported AAEs than 
Table 2. Acute adverse events (AAEs) to MR groups.

\begin{tabular}{|c|c|c|c|}
\hline \multirow{2}{*}{ Symptoms } & \multicolumn{3}{|c|}{ MR groups } \\
\hline & Contrast enhanced $n=259$ & Control $n=434$ & P-value \\
\hline No. of patients with AAEs & $40(15.4 \%)$ & $32(7.4 \%)$ & $<0.001$ \\
\hline No. of AAEs observed & 48 (18.5\%) & $39(9.0 \%)$ & $<0.001$ \\
\hline \multicolumn{4}{|l|}{ Type of AAEs } \\
\hline \multicolumn{4}{|l|}{ Mild } \\
\hline Nausea & $10(3.9 \%)$ & $7(1.6 \%)$ & 0.078 \\
\hline Dizziness & $19(7.3 \%)$ & $13(3 \%)$ & 0.014 \\
\hline Vomiting & $1(0.4 \%)$ & 0 & 0.375 \\
\hline Urticaria & $1(0.4 \%)$ & 0 & 0.375 \\
\hline Itching & 0 & 0 & \\
\hline Cough & $1(0.4 \%)$ & $1(0.2 \%)$ & 1 \\
\hline \multicolumn{4}{|l|}{ Moderate } \\
\hline Severe vomiting & 0 & 0 & \\
\hline Marked urticaria & 0 & 0 & \\
\hline Breathing difficulties & 0 & 0 & \\
\hline Bronchospasm & 0 & 0 & \\
\hline Facial/Laryngeal edema & 0 & 0 & \\
\hline Vasovagal attack & 0 & 0 & \\
\hline \multicolumn{4}{|l|}{ Severe } \\
\hline Hypotensive shock & 0 & 0 & \\
\hline Respiratory arrest & 0 & 0 & \\
\hline Cardiac arrest & 0 & 0 & \\
\hline Convulsion & 0 & 0 & \\
\hline Other & $16(6.2 \%)$ & $18(4.2 \%)$ & 0.276 \\
\hline Fatigue & $2(1.3 \%)$ & $4(0.9 \%)$ & 1 \\
\hline Fever & 0 & $1(0.2 \%)$ & 1 \\
\hline Headache & $10(3.9 \%)$ & $11(2.5 \%)$ & 0.363 \\
\hline Back pain & 0 & $1(0.2 \%)$ & 1 \\
\hline Abdominal pain & $1(0.4 \%)$ & $1(0.2 \%)$ & 1 \\
\hline Sweating & $1(0.4 \%)$ & 0 & 0.375 \\
\hline Stinging in the lower region & $1(0.4 \%)$ & 0 & 0.375 \\
\hline Heatsensation & $1(0.4 \%)$ & 0 & 0.375 \\
\hline
\end{tabular}

patients undergoing CT. This could be due to several reasons: 1) CT scans are usually fast, while MR scans run for 30 min or more. In a study by Thomsen [9], the noise from a MR scan gradients have been mentioned as no patient reported headache after being scanned in an almost silent $0.1 \mathrm{~T}$ MR scanner, whereas more than $10 \%$ of the patients in another study being scanned in a noisy 1.5T MR scanner reported headache [13]. In our study, $3.9 \%$ in contrast enhanced MR and $2.5 \%$ in the MR control group reported headache, and none in the CT groups. Claustrophobia and a number of other psychological problems, including depression, anxiety and panic disorders may be encountered by as many as $5 \%$ - $10 \%$ of patients undergoing MRI [14]. 2) Furthermore, dizziness was reported more frequently in the contrast enhanced MR group than in the MR control and CT groups. Since 50\% of the contrast enhanced MR examinations performed were of head indicating cerebral disease, this could be a factor. 3) The control groups included for both MR and CT differed in type of imaging. In the CT control group 1.9\% of the patients reported an AAE while $7.4 \%$ in the MR control group did so. Although the patients in the two groups underwent different type of imaging because of different clinical indications or underlying diseases, this 
Table 3. Acute adverse events (AAEs) to CT groups.

\begin{tabular}{|c|c|c|c|}
\hline \multirow{2}{*}{ Symptoms } & \multicolumn{3}{|c|}{ CT groups } \\
\hline & Contrast enhanced $n=259$ & Control $n=434$ & P-value \\
\hline No. of patients with AAEs & $26(5.8 \%)$ & $4(1.9 \%)$ & 0.026 \\
\hline No. of AAEs observed & $35(7.7 \%)$ & $5(2.3 \%)$ & 0.003 \\
\hline \multicolumn{4}{|l|}{ Type of AAEs } \\
\hline \multicolumn{4}{|l|}{ Mild } \\
\hline Nausea & $8(1.7 \%)$ & $1(0.4 \%)$ & 0.284 \\
\hline Dizziness & $13(2.9 \%)$ & $2(0.9 \%)$ & 0.162 \\
\hline Vomiting & 0 & 0 & \\
\hline Urticaria & 0 & 0 & \\
\hline Itching & $1(0.2 \%)$ & $1(0.5 \%)$ & 0.542 \\
\hline Cough & $1(0.2 \%)$ & 0 & 1 \\
\hline \multicolumn{4}{|l|}{ Moderate } \\
\hline Severe vomiting & 0 & 0 & \\
\hline Marked urticaria & $1(0.2 \%)$ & 0 & 1 \\
\hline Breathing difficulties & 0 & 0 & \\
\hline Bronchospasm & 0 & 0 & \\
\hline Facial/Laryngeal edema & 0 & 0 & \\
\hline Vasovagal attack & 0 & 0 & \\
\hline \multicolumn{4}{|l|}{ Severe } \\
\hline Hypotensive shock & 0 & 0 & \\
\hline Respiratory arrest & 0 & 0 & \\
\hline Cardiac arrest & 0 & 0 & \\
\hline Convulsion & 0 & 0 & \\
\hline Other & $11(2.4 \%)$ & $1(0.5 \%)$ & .116 \\
\hline Fever & $1(0.2 \%)$ & 0 & 1 \\
\hline Back pain & $1(0.2 \%)$ & 0 & 1 \\
\hline Burning sensation & $2(0.4 \%)$ & 0 & 1 \\
\hline Swollen arm & $1(0.2 \%)$ & 0 & 1 \\
\hline Fatigue & $1(0.2 \%)$ & 0 & 1 \\
\hline Taste sensation & $1(0.2 \%)$ & 0 & 1 \\
\hline Visual disturbance & $1(0.2 \%)$ & 0 & 1 \\
\hline Tingling on tongue & $1(0.2 \%)$ & 0 & 1 \\
\hline Tingling in fingers & 0 & $1(0.5 \%)$ & 0.324 \\
\hline Tingling on cheeks & $1(0.2 \%)$ & 0 & 1 \\
\hline Chest pain & $1(0.2 \%)$ & 0 & 1 \\
\hline
\end{tabular}

cannot explain the difference alone. This underlines once again that one should be cautious before claiming that the contrast media was the cause of an AAE.

Overall, AAEs were significantly higher in the contrast enhanced groups compared to control groups for both MR and CT groups. The most frequent AAEs in both the MR and CT groups were nausea and dizziness. Dizziness occurred more frequently in the contrast enhanced MR than in the control group $(\mathrm{P}<0.014)$; MR of the brain indicating cerebral disease was nearly only performed in the contrast group. Therefore, it could also be a consequence of the disease, and not an adverse event to contrast medium. Although nausea also occurred frequently, the difference was not statistically significant. The same were observed in the CT groups. Nausea and dizziness were the two AAEs reported the most, but none of them achieved statistical significance. Itching also occurred once in both CT groups, but with no statistical 
significance. In a prospective study of 14.299 patients who received a macrocyclic gadolinium chelat, one of the most reported adverse reaction was nausea $(0.25 \%)$ [15]. Other retrospective studies [16-18] also reported nausea as one of the most frequent mild AAEs to MR contrast medium. Hunt et al. reported retrospectively hives (52.5\%) and nausea (17.6\%) as the most common adverse reaction to iodinated and gadolinium contrast media in 456.930 doses [12]. Herborn et al. [19] and Ishiguchi et al. [20] investigated prospectively the same MR contrast medium as we used in our study, and they both reported nausea ( 0.17 and 0.41 , respectively) followed by vomiting ( 0.05 and 0.09 , respectively) as the most common reported adverse events to gadoterate meglumine. This is in agreement with our study, although vomiting was only reported once in the contrast enhanced MR group and marked urticaria was reported once in contrast enhanced CT group. Our study only included outpatients, whereas Hunt et al. [12] and Ishiguchi et al. [20] both included inpatients as well. Inpatients may have reported reactions caused by their underlying disease and not by the administration of contrast media, since they in general are sicker than outpatients. None of the abovementioned studies included a control group. In a prospective study by Schild et al. [4] of the incidence of AAEs to iodine-based contrast medium, both nausea (2.4\%) and dizziness (1.3\%) were more frequent reported than other AAEs in the contrast group. However, the same adverse events were also reported in the control group, $(0.3 \%)$ and (2\%) respectively. This shows that both nausea and dizziness also occurs in the groups not receiving contrast media. In a review by Dooley et al. [21], the overall incidence of AAEs and discomfort (metal taste, warmth, local pain etc.) of CT contrast medium iomeprol ranged from $3 \%-49.7 \%$. Among the pseudoallergic reactions nausea was the most common, $1.3 \%$. In our study, it was $1.7 \%$. It is difficult to draw a clear conclusion about the incidence of AAEs following gadolinium administration, as the studies differ in design and definition of AAEs. Davenport et al. [22] and Abujudeh et al. [17] both reported the incidence to be $0.15 \%$ and $0.16 \%$, respectively. Both studies were retrospective and may underestimate the actual rate. In a prospective post marketing surveillance study by Herborn et al. [19] of 24,308 patients who received MR contrast media intravenously, the incidence reported was higher, $0.4 \%$. Another prospective study by Ishigushi et al. [20] of 3444 patients, the incidence was $0.93 \%$. The incidences reported in these prospective studies are higher than for the retrospective studies. The prospective studies of Herborn et al. [19] and Ishigushi et al. [20] used gadoterate meglumine, the same contrast media as used in our study but they did not use active interview to record AAEs. The high incidence of AAEs reported in our contrast enhanced MR group (18.5\%) may be due to that we used active interview to record AAEs. Nevertheless, Brugieres et al. [23] did report in a prospective study an incidence of $17.3 \%$ after gadoterate meglumine administration.

Nearly all AAEs reported in both the MR and CT groups were categorized as mild, and did not require any treatment. Moderate AAEs were reported in one patient in the contrast enhanced CT group. None of the AAEs were severe. The findings in our study supports the results presented in other studies that evaluated both gadolinium and iodinated contrast media $[4,5,12,18]$.

The strength of our study was that it prospectively evaluated AAEs comparing both contrast enhanced MR and CT groups with control groups. A structured questionnaire was used interviewing all 1358 patients. Furthermore, our study included only outpatients not exposed to contrast media 30 days before the actual examination. To our knowledge, only one [4] prospective observational study on AAEs has been performed in outpatients compared to a control group; it is nearly always inpatients which one has better access to, but nowadays most patients undergoing CT and MR are outpatients. Our results were comparable between groups, because all patients underwent exactly the same observation; it was a single center study. The limitations in this study were 1) the number of patients, although it took nearly two and a half years for one person to recruit the 1358 patients. The number of patients in the groups varies with the MR control group and the contrast enhanced CT including more than 400 patients. It was easier to recruit to those groups simply because they are frequent in the department. In our department around 2/3 of the patients undergoing CT undergo contrast enhanced CT and for MR only 1/3 undergo contrast enhanced MR. We strictly followed the routine of the department for use of contrast medium. The decision to administer contrast medium to the patients was not influenced by this study. The patients received what was clinically indicated. 2) the inclusion criteria were rather strict (only outpatients, no contrast medium 30 days or surgery 30 days within the imaging), and 3) demographic differences between the groups. It would have been unethical to give the patients in the control groups saline. In principle it could have been done with the patients undergoing unenhanced imaging, but then we would have exposed $50 \%$ of the patients in the control group to unneeded contrast medium. Also we could not allow that $50 \%$ of the patients scheduled for a contrast enhanced examination not to receive contrast medium, as we then might overlook lesions.

\section{CONCLUSION}

Incidences of AAEs were significantly higher in the enhanced groups compared to control groups for both MR 
and CT, but the same type of AAEs were seen in the control groups as in the contrast groups. The incidences vary a lot from study to study; the circumstances (retrospective, prospective, active interview, structured questionnaire, simple question, noise, patient information) may explain the variations to some extent. All events except one were categorized as mild. The fact that the same AAEs are seen in both after contrast and no contrast medium administration raises the question whether a mild adverse event after exposure to contrast medium should be classified as an adverse event to the contrast medium in all situations. An incorrect registration may have impact on future imaging, and may lead to excessive testing of the patients and thereby waste of the scarce resources.

\section{ACKNOWLEDGEMENTS}

The authors would like to thank the staff who participated in this study. We are very grateful for the financial support of the following sponsors: Johannes Fogs Fond and Aase og Ejnar Danielsens Fond.

\section{REFERENCES}

[1] Thomsen, H.S. and Webb, J. (2012) The Lalli and Weber effects and the incidence of acute non-renal adverse reactions to contrast media. Acta Radiologica, 53, 953-954. doi:10.1258/ar.2012.12a006

[2] Lalli, A.F. (1980) Contrast media reactions: Data analysis and hypothesis. Radiology, 134, 1-12.

[3] Weber, J.C.P. (1984) Epidemiology of adverse reactions to nonsteroidal anti-inflammatory drugs. In: Rainsford, K.D. and Velo, G.D., Eds., Side-effects of anti-inflammatory drugs, advances in inflammation research. Raven Press, New York, 1-7.

[4] Schild, H.H., Kuhl, C.K., Hübner-Steiner, U., Böhm, I. and Speck, U. (2006) Adverse events after unenhanced and monomeric and dimeric contrast-enhanced CT: A prospective randomized controlled trial. Radiology, 240, 56-64. doi:10.1148/radiol.2393050560

[5] Dillman, J.R., Ellis, J.H., Cohan, R.H., Strouse, P.J. and Jan, S.C. (2007) Frequency and severity of acute allergiclike reactions to gadolinium-containing IV contrast media in children and adults. AJR American Journal of Roentgenology, 189, 1533-1538. doi:10.2214/AJR.07.2554

[6] Murphy, K.J., Brunberg, J.A. and Cohan, R.H. (1996) Adverse reactions to gadolinium contrast media: A review of 36 cases. AJR American Journal of Roentgenology, 167, 847-849. doi:10.2214/ajr.167.4.8819369

[7] Knopp, M.V., Balzer, T., Esser, M., Kashanian, F.K., Paul, P. and Niendorf, H.P. (2006) Assessment of utilization and pharmacovigilance based on spontaneous adverse event reporting of gadopentate dimeglumine as a magnetic resonance contrast media after 45 million administrations and 15 years of clinical use. Investigative Radiology, 41, 491-499. doi:10.1097/01.rli.0000209657.16115.42

[8] Morcos, S.K. and Thomsen, H.S. (2001) Adverse reac- tions to iodinated contrast media. European Radiology, 11, 1267-1275. doi:10.1007/s003300000778

[9] Thomsen, H.S. (1997) Frequency of acute adverse events to a non-ionic low-osmolar contrast medium: The effect of verbal interview. Pharmacology \& Toxicology, 80, 108-110. doi:10.1111/j.1600-0773.1997.tb00292.x

[10] Niendorf, H.P., Dinger, J.C., Haustein, J., Cornelius, I., Alhassan, A. and Clauss, W. (1991) Tolerance data of GdDTPA: A review. European Journal of Radiology, 13, 15-20. doi:10.1016/0720-048X(91)90049-2

[11] Nelson, K.L., Gifford, L.M., Lauber-Huber, C., Gross, C.A. and Lasser, T.A. (1995) Clinical safety of gadopentetate dimeglumine. Radiology, 196, 439-443.

[12] Hunt, C.H., Hartman, R.P. and Hesley, G.K. (2009) Frequency and severity of adverse effects of iodinated and gadolinium contrast materials: Retrospective review of 456,930 doses. AJR American Journal of Roentgenology, 193, 1124-1127. doi:10.2214/AJR.09.2520

[13] Goldstein, H.A., Kashanian, F.K., Blumettei, R.F., Holyoak, W.L., Hugo, F.P. and Blumenfield, D.M. (1990) Safety assessment of gadopentetate dimeglumine in US clinical trials. Radiology, 174, 17-23.

[14] Shellock, F.G., Morisoli, S.M. and Ziarati, M. (1994) Measurement of acoustic noise during MR imaging: Evaluation of six "Worst-Case” pulse sequences. Radiology, 191, 91-93.

[15] Forsting, M. and Palkowitsch, P. (2010) Prevalence of Acute adverse events to gadobutrol-A highly concentrated macrocyclic gadolinium chelate: Review of 14,299 patients from observational trials. European Journal of Radiology, 74, e186-e192. doi:10.1016/j.ejrad.2009.06.005

[16] Bruder, O., Schneider, S., Nothnagel, D., Pilz, G., Lombardi, M., Sinha, A., Wagner, A., Dill, T., Frank, H, van Rossum, A., Schwitter, J., Nagel, E., Senges, J., Sabin, G., Sechtem, U. and Mahrholdt, H. (2011) Acute adverse events to gadolinium-based contrast agents in CMR: multicenter experience with 17,767 patients from the Euro CMR Registry. JACC Cardiovasc Imaging, 4, 1171-1176. doi:10.1016/j.jcmg.2011.06.019

[17] Abujudeh, H.H., Kosaraju, V.K. and Kaewlai, R. (2010) Acute adverse events to gadopentetate dimeglumine and gadobenate dimeglumine: Experience with 32,659 injections. AJR American Journal of Roentgenology, 194, 430 434. doi:10.2214/AJR.09.3099

[18] Li, A., Wong, C.S., Wong, M.K., Lee, C.M. and Au Yeung, M.C. (2006) Acute adverse events to magnetic resonance contrast media: Gadolinium chelates. British Journal of Radiology, 79, 368-371. doi:10.1259/bjr/88469693

[19] Herborn, C.U., Honold, E., Wolf, M., Kemper, J., Kinner, S., Adam, G. and Barkhausen, J. (2007) Clinical safety and diagnostic value of the gadolinium chelate gadoterate meglumine (Gd-DOTA). Investigative Radiology, 42, 5862. doi:10.1097/01.rli.0000248893.01067.e5

[20] Ishiguchi, T. and Takahashi, S. (2010) Safety of gadoterate meglumine (Gd-DOTA) as a contrast agent for magnetic resonance imaging: Results of a post-marketing surveillance study in Japan. Drugs $R D$, 10, 133-145. doi:10.2165/11539140-000000000-00000 
[21] Dooley, M. and Jarvis, B. (2000) Iomeprol: A review of its use as a contrast medium. Drugs, 59, 1169-1186. doi:10.2165/00003495-200059050-00013

[22] Davenport, M.S., Dillman, J.R., Cohan, R.H., Hussain, H.K., Khalatbari, S., McHugh, J.B. and Ellis, J.H. (2013) Effect of abrupt substitution of gadobenate dimeglumine for gadopentetate dimeglumine on rate of allergic-like reactions. Radiology, 266, 773-782. doi:10.1148/radiol.12120253

[23] Brugières, P., Gaston, A., Degryse, H.R., Parizel, P.M., de Schepper, A.M., Berry, I., Manelfe, C., Le Bras, F., Marsault, C., Wichmann, W. and Valavanis, A. (1994) Randomised double blind trial of the safety and efficacy of two gadolinium complexes (Gd-DTPA and Gd-DOTA). Neuroradiology, 36, 27-30. doi:10.1007/BF00599189 